\title{
Markedly Increased Small Dense Low-Density Lipoprotein During Acute Phase in Childhood and Adolescent Nephrotic Syndrome
}

Keisuke Sugimoto ( $\nabla$ ksugimo@med.kindai.ac.jp )

Kindai University Faculty of Medicine https://orcid.org/0000-0002-0978-7511

Kohei Miyazaki

Kinki Daigaku Igakubu Daigaku Byoin

Takuji Enya

Kinki Daigaku Igakubu Daigaku Byoin

Tomoki Miyazawa

Kinki Daigaku Igakubu Daigaku Byoin

Yuichi Morimoto

Kinki Daigaku Igakubu Daigaku Byoin

Rina Ohshima

Kinki Daigaku Igakubu Daigaku Byoin

Tsukasa Takemura

Kushimoto municipality hospital

Mitsuru Okada

Kinki Daigaku Igakubu Daigaku Byoin

\section{Research article}

Keywords: Hyperlipidemia, low-density lipoprotein, nephrotic syndrome, childhood adolescence

Posted Date: August 18th, 2020

DOI: https://doi.org/10.21203/rs.3.rs-55523/v1

License: (c) (i) This work is licensed under a Creative Commons Attribution 4.0 International License.

Read Full License 


\section{Abstract}

Background: Hyperlipidemia is an important characteristic feature of idiopathic nephrotic syndrome (NS) in children. This study was conducted to examine the lipid profiles, including small dense low-density lipoprotein (sdLDL-C), in childhood-onset NS.

Methods: This retrospective study enrolled patients diagnosed with initial-onset NS in childhood and adolescence. Study parameters included lipid profiles. The "alternative LDL window" comprises the number and sizes of LDL particles estimated according to non-HDL-C and TG levels.

Results: A total of 39 patients were enrolled who exhibited markedly increased lipid abnormalities, including TC, TG, LDL-C, and non-HDL-C levels (TC, 409.7 TC, TG, and sizes of LDL particles estimated as non-HDL-C, 332.3). Of the 39 patients, 32 (82\%) were categorized in the area of hyper-TG/-non-HDL levels, which is considered as sdLDL. A positive correlation was found between non-HDL-C and TC $(r=0.96, P<$ 0.001), TG $(r=0.38, P=0.018), L D L-C(r=0.84, P<0.001), T C / H D L(r=0.53, P<0.001)$, and atherogenic index of plasma $(r=0.42, P=0.008)$.

Conclusions: Our study demonstrated markedly increased lipid profiles during the acute phase of NS. Evaluation of lipid profiles using the "alternative LDL window" may help understand the state of hyperlipidemia in NS.

\section{Background}

Hyperlipidemia is an important characteristic feature of idiopathic nephrotic syndrome (NS) in children $[1-3]$. It can increase permeability to albumin, resulting in proteinuria via oxidant stress $[4,5]$. Hyperlipidemia gradually tends to normalize with remission, although it remains after remission in some patients. Low-density lipoprotein (LDL) apheresis used as a treatment for patients with resistant NS supports that dyslipidemia, including high levels of LDL cholesterol (LDL-C), is associated with the development and risk factors of NS [6, 7].

Hirano et al. developed an assay for the quantification of cholesterol and separated LDL-C particles into three or four major LDL subclasses based on density [8]. This assay is not widely used in general clinical practice due to the difficulty, especially in children, to obtain sufficient blood samples. Subsequently, Hayashi et al. proposed a simpler method, the "alternative LDL window," that uses conventional methods for estimating high small dense LDL-C (sdLDL-C) levels [9]. sdLDL-C level was found to be increased in patients combined with hyperlipidemia [10]. Thus, the level of sdLDL-C is a candidate for a novel risk factor, or a risk marker for atherosclerotic vascular complications such as coronary heart disease $[10,11$, 12]. sdLDL is also currently considered as one of the lipoproteins that leads to the development of cardiovascular disease (CVD) in patients with chronic kidney disease (CKD) [13]. Canden et al. reported that subclinical CVD and its association with risk factors were independently associated only with higher LDL-C levels in children with steroid-resistant nephrotic syndrome (SRNS) [14]. However, no evaluation of sdLDL-C levels in NS was performed. Therefore, this study was conducted to examine the cholesterol 
composition, including sdLDL-C level in NS referring to the "alternative LDL window," to provide a better understanding of the lipid profiles of childhood-onset NS.

\section{Methods}

This retrospective study enrolled patients diagnosed with initial-onset NS in childhood and adolescence from April 2010 to March 2020. All patients were recruited from the Department of Pediatrics at Kindai University Hospital, where they were treated with prednisolone according to the recommendations of the Japanese Society of Pediatric Nephrology. Remission was defined as no evidence of proteinuria in earlymorning specimens for 3 consecutive days. Patients with NS caused due to other renal diseases and those with familial hyperlipidemia, receiving lipid-lowering drugs, and lack of sufficient data at the time of enrollment were excluded. At the point of study entry, the height and weight [for body mass index (BMI) calculation] were measured. Study parameters were estimated before the initiation of prednisolone. Laboratory data consisting of serum creatinine, estimated GFR, serum concentrations of total protein (TP), albumin (Alb), total cholesterol (TC), triglyceride (TG), LDL-C, HDL-cholesterol (HDL-C), uric acid, and creatinine levels were examined. Non-HDL-C level is calculated using the following formula: [non-HDL-C $(\mathrm{mg} / \mathrm{dL})=\mathrm{TC}-\mathrm{HDL}-\mathrm{C}]$. The concentrations of urinary protein and creatinine were also measured. The baseline of lipid profiles was defined based on the National Heart, Lung, and Blood Institute (NHLBI) guidelines, which recommend universal screening of children for hyperlipidemia [15]. The average of atherogenic index of plasma (AIP) was calculated using the formula log10 (TG/HDL-C) [16].

\section{LDL particle size and LDL window determination}

The "alternative LDL window" comprises the number and sizes of LDL particles estimated according to the plasma non-HDL-C and TG levels. Non-HDL-C is a surrogate marker for apoB [17]; therefore, it was used instead of apoB. Non-HDL-C level was calculated by subtracting the HDL-C level from the total-C level. Subjects were divided into the following four groups: normal (non-HDL-C $<170$ and TG $<150$ $\mathrm{mg} / \mathrm{dL}$ ), hyper-TG (non-HDL-C $<170$ and TG $\geq 150 \mathrm{mg} / \mathrm{dL}$ ), hyper-non-HDL (non-HDL-C $\geq 170$ and $\mathrm{TG}<$ $150 \mathrm{mg} / \mathrm{dL}$ ), and hyper-TG/-non-HDL (non-HDL-C $\geq 170$ and TG $\geq 150 \mathrm{mg} / \mathrm{dL}$ ).

\section{Statistical analysis}

Quantitative data are presented as mean \pm standard deviation (SD). Pearson's correlation coefficients were used to analyze the correlations between variables. A P value of $<0.05$ was considered as statistically significant.

\section{Results}

A total of 87 patients were included, of whom 39 were enrolled for the final analysis in this study after following the exclusion criteria. Baseline characteristics and clinical data, including the lipid profiles, of the subjects are shown in Table 1. The median age at onset was 6.3 years (range 0.7-17.1 years), and the male-to-female ratio was 20:19. Five patients were steroid-resistant. Subsequent renal biopsy 
disclosed focal glomerular sclerosis in 3 patients. Lipid abnormalities, including TC, TG, LDL-C, and nonHDL-C levels, were significantly higher than the baseline according to the NHLBI guidelines (TC, $409.7 \pm$ 109.8; TG, $241.0 \pm 147.6$; LDL-C, $297.8 \pm 126.6$; non-HDL-C, $332.3 \pm 113.3$ ), whereas HDL-C levels was below $40 \mathrm{mg} / \mathrm{dL}$ in 2 patients. Of the 39 patients, 32 (82\%) exhibited hyper-TG/-non-HDL levels, and 3 (8\%) patients presented with hyper-non-HDL levels. Four patients presented with normal TG and non-HDL levels (Fig. 1). The results of Pearson's correlation analyses demonstrated that non-HDL-C level positively and significantly correlated with the levels of TC $(r=0.96, P<0.001), T G(r=0.38, P=0.018), L D L-C(r=$ $0.84, P<0.001), T C / H D L(r=0.53, P<0.001)$, and AIP $(r=0.42, P=0.008)$ and negatively correlated with TP $(r=-0.62, P<0.001)$ and Alb $(r=-0.66, P<0.001)$ levels. The AIP level was $0.46 \pm 0.39$ and more than 0.24 in 26 patients $(69 \%)$. The results of Pearson's correlation analyses revealed that AIP level positively and significantly correlated with the levels of TG $(r=0.82, P<0.001), L D L-C(r=0.39, P=0.01), T C / H D L(r$ $=0.81, P<0.001)$, and UP/Crn $(r=0.43, P=0.006)$ and negatively correlated with onset age $(r=-0.55, P<$ $0.001)$, weight $(r=-0.46, P=0.003)$, TP $(r=-0.66, P<0.001)$, Alb $(r=-0.56, P<0.001)$, and HDL-C $(r=-$ $0.78, P<0.001)$.

\section{Discussion}

It is well known that TC, TG, and LDL-C levels are markedly increased during the nephrotic state. Especially in the initial onset, dyslipidemia appears frequently compared to that during the relapse phase. The magnitude of dyslipidemia in NS is considered to be directly associated with the severity of proteinuria [18]. In our study, significant dyslipidemia with not only high levels of TC, TG and LDL-C, but also non-HDL levels were noted. Non-HDL-C exhibits several benefits beyond LDL-C and can be used for lipid management when a patient's samples show a TG level of $>400 \mathrm{mg} / \mathrm{dL}$ or non-fasting state [19]. As unifying the fasting samples at the onset is difficult, evaluating non-HDL-C level is considered to be useful, especially in children. Several studies reported high non-HDL-C levels in patients with metabolic syndrome. In our study, the non-HDL-C level of patients with NS was significantly higher than previously reported data [20, 21]. Furthermore, we focused on the LDL particle size to evaluate non-HDL-C level. TG is a powerful inverse determinant of LDL particle size $[9,17]$. In other words, the higher the TG value, the smaller the particle size of LDL, which is recognized as sdLDL. Using the "alternative LDL window," understanding the condition of dyslipidemia at a glance in the acute phase of NS is easy. When patients belong to the area of hyper-TG/-non-HDL, it implies that among those patients with sdLDL, $82 \%$ with hyper-TG/-non-HDL levels could be exposed to the risk of endothelial cell damage.

Endothelial cell damage contributes to the enhancement of vascular permeability, and it is considered to be one of the important factors in the development of NS. Sharma et al. reported that patients with SRNS exhibited high levels of endothelial dysfunction markers [22]. sdLDL is a lipoprotein that strongly induces arteriosclerosis and is closely associated with cardiovascular events. Using these criteria, the majority of our patients were found exhibit a considerable risk of developing premature vascular disease. Hyperlipidemia is also involved in the pathogenesis of various acute complications in NS [23, 24]. Interestingly, Ambrosch et al. provided novel clues on the atherogenic mechanisms of sdLDL, which sensitize vascular cells to inflammatory signals more effectively than normal-sized LDL particles [25]. 
The oxidative modification of LDL plays a vital role in the pathogenesis of several vascular diseases [26, 27]. In addition, endothelial dysfunction and inflammation occur in even an asymptomatic proteinuria state [28]. AIP is used to predict the angiographic complexity of peripheral artery disease, and it has been confirmed to be significantly correlated with other important atherosclerosis indexes such as LDL-C particle size and sdLDL-C $[16,29]$. It was classified into 3 risk levels for CVD according to the following values: -0.3 to 0.1 for low risk, $0.1-0.24$ for medium risk, and $>0.24$ for high risk [30]. In our study, $69 \%$ of the patients exhibited high levels of AIP, which implies they may exhibit a high-risk condition during the acute phase, although it may be a short period. Clinically, no obvious complications were observed in both acute and remission phases in our study. This is not surprising as children basically do not present with an underlying illness and may demonstrate fewer complications than adults. In our study, we found that AIP positively and significantly correlated with lipid profiles such as TG, LDL-C, and TC/HDL negatively correlated with TP and Alb. The latter findings generally normalize, and hyperlipidemia also tends to disappear with the resolution of proteinuria. However, some studies reported persisting lipid anomalies during remission [31, 32]. In one study of 30 patients with childhood-onset steroid-sensitive NS (SSNS), dysregulation of lipids, including increased TC levels, and LDL-C were persistent during the follow-up at 4-15 years after the completion of steroid therapy [33]. The hyperlipidemic profiles remained despite the remission, especially in frequently relapsing NS cases [31]. Furthermore, subclinical cardiovascular disease and its association with risk factors are detected in children with SRNS [14]. In addition, lowering cholesterol levels using HMG-CoA reductase inhibitors during childhood may reduce the risk for atherosclerotic changes [34]. Hence, lipid-lowering agents may be of benefit in certain patients with NS such as those with SRNS.

Currently, the number of young patients improving from pediatric to adult renal care progressively increased due to improved management worldwide. It appears possible that even SSNS, which was previously believed to be entirely benign, may engender an increased risk for hypertension, atherosclerosis, and CKD in adulthood [35]. In our study, no significant correlation existed between the data of lipid profiles and eGFR. However, the CKD in Children Study reported that the presence of dyslipidemia was associated with the reduction of eGFR in patients with nonglomerular disease [36].

The limitations of this study include the small number of patients. Further studies are required to examine the changes in the levels of lipid profiles in individuals at the remission phase, which could help predict the severity and prognosis. In addition to increasing the number of patients, considering the measurement of aortic pulse wave velocity, carotid intima media thickness, and left ventricular mass is necessary to evaluate the risk factors of cardiovascular disease.

\section{Conclusion}

Clinically, lipid profiles such as TC and TG are routinely evaluated in patients with NS. De Silva et al recommended that the baseline lipid profile, including TC, TG, HDL-C, and LDL-C, should be evaluated in children with SRNS [37]. Our results also propose the evaluation of lipid profiles and the need for careful 
characterization of patients. The "alternative LDL window" and AIP are relatively easy to obtain using routine biochemical parameters and help understand the state of hyperlipidemia in patients with NS.

\section{Abbreviations}

AIP; atherogenic index of plasma, Alb; albumin, BMl; body mass index, CKD; chronic kidney disease, CVD; cardiovascular disease, HDL-cholesterol (HDL-C)LDL; Low-density lipoprotein, LDL-C; low-density lipoprotein choresterol, NHLBl; National Heart, Lung, and Blood Institute, NS; nephrotic syndrome, sdLDLC; Small dense low-density lipoprotein, SRNS; steroid-resistant nephrotic syndrome, TC; Total cholesterol, TG; Triglyceride,

\section{Declarations}

\section{Ethics approval and consent to participate}

The Institutional Ethics Committee of the Faculty of Medicine of Kindai University approved this study (2020-065). Consent was obtained from a parent or guardian on behalf of any participants under the age of 16 . The study was conducted in accordance with the ethical principles of the Declaration of Helsinki.

\section{Consent to publish}

All authors, patient and her parent consented for this publication.

\section{Availability of data and materials}

The datasets used and/or analysed during the current study are available from the corresponding author on reasonable request.

\section{Competing interest}

The authors declare that they have no competing interest.

\section{Funding}

This study was not received any funding.

\section{Author's Contributions}

$\mathrm{KS}$ and $\mathrm{MO}$ conceived and designed the study. KM, TE, TM and RO carried out participant enrollment and managed data collection. TT led data analysis and specimen management and wrote the manuscript. TT provided guidance and expertise on data analysis and interpretation. YM assisted in data analysis and manuscript preparation. All authors contributed to the final manuscript.

\section{Acknowledgments}


No grant support was received for this study. The authors would like to thank Enago (www.enago.jp) for the English language review.

\section{References}

1. Attman PO, Alaupovic P. Pathogenesis of hyperlipidemia in the nephrotic syndrome. Am J Nephrol. 1990;10:69-75.

2. Thabet MA, Salcedo JR, Chan JC. Hyperlipidemia in childhood nephrotic syndrome. Pediatr Nephrol. 1993;7:559-66.

3. Agrawal S, Zaritsky JJ, Fornoni A, Smoyer WE. Dyslipidaemia in nephrotic syndrome: mechanisms and treatment. Nat Rev Nephrol. 2018;14:57-70.

4. Musante L, Candiano G, Petretto A, Bruschi M, Dimasi N, Caridi G, Pavone B, Del Boccio P, Galliano M, Urbani A, Scolari F, Vincenti F, Ghiggeri GM. Active focal segmental glomerulosclerosis is associated with massive oxidation of plasma albumin. J Am Soc Nephrol. 2007;18:799-810.

5. Ruan XZ, Varghese Z, Moorhead JF. An update on the lipid nephrotoxicity hypothesis. Nat Rev Nephrol. 2009;5:713-21.

6. Muso E. Beneficial effect of LDL-apheresis in refractory nephrotic syndrome. Clin Exp Nephrol. 2014;18:286-90.

7. Hari P, Khandelwal P, Smoyer WE. Dyslipidemia and Cardiovascular Health in Childhood Nephrotic Syndrome. Pediatr Nephrol. 2019;35:1601-19.

8. Hirano T, Ito $Y, K o b a ~ S$, et al. Clinical significance of small dense low-density lipoprotein cholesterol levels determined by the simple precipitation method. Arterioscler Thromb Vasc Biol. 2004;24:558-63.

9. Hayashi T, Koba S, Ito Y, Hirano T. Method for estimating high sdLDL-C by measuring triglyceride and apolipoprotein B levels. Lipids Health Dis. 2017;16:21.

10. Hoogeveen RC, Gaubatz JW, Sun W, Dodge RC, Crosby JR, Jiang J, Couper D, Virani SS, Kathiresan S, Boerwinkle E, Ballantyne CM. Small dense low-density lipoprotein-cholesterol concentrations predict risk for coronary heart disease: the Atherosclerosis Risk In Communities (ARIC) study. Arterioscler Thromb Vasc Biol. 2014;34:1069-77.

11. Sakai K, Koba S, Nakamura Y, Yokota Y, Tsunoda F, Shoji M, Itoh Y, Hamazaki Y, Kobayashi Y. Small dense low-density lipoprotein cholesterol is a promising biomarker for secondary prevention in older men with stable coronary artery disease. Geriatr Gerontol Int. 2018;18:965-72.

12. Hsu SH, Jang MH, Torng PL, Su TC. Positive Association Between Small Dense Low-Density Lipoprotein Cholesterol Concentration and Biomarkers of Inflammation, Thrombosis, and Prediabetes in Non-Diabetic Adults. J Atheroscler Thromb. 2019;26:624-35.

13. Shen $\mathrm{H}$, et al. Small dense low-density lipoprotein cholesterol was associated with future cardiovascular events in chronic kidney disease patients. BMC Nephrol. 2016;17:143.

14. Candan C, Canpolat N, Gökalp S, Yıldız N, Turhan P, Taşdemir M, Sever L, Çalışkan S. Subclinical cardiovascular disease and its association with risk factors in children with steroid-resistant 
nephrotic syndrome. Pediatr Nephrol. 2014;29:95-102.

15. Expert Panel on Integrated Guidelines for Cardiovascular Health and Risk Reduction in Children and Adolescents; National Heart, Lung, and Blood Institute. Expert panel on integrated guidelines for cardiovascular health and risk reduction in children and adolescents: summary report. Pediatrics. 2011;128 Suppl 5:213-56.

16. Dobiasova M, Frohlich J. The plasma parameter log (TG/HDL-C) as an atherogenic index: correlation with lipoprotein particle size and esterification rate in apoB-lipoprotein-depleted plasma (FER(HDL)). Clin Biochem. 2001;34:583-8.

17. Austin MA, King MC, Vranizan KM, Krauss RM. Atherogenic lipoprotein phenotype. A proposed genetic marker for coronary heart disease risk. Circulation. 1990;82:495-506.

18. Vaziri ND. Disorders of lipid metabolism in nephrotic syndrome: mechanisms and consequences. Kidney Int. 2016;90:41-52.

19. Teramoto T, Sasaki J, Ishibashi S, Birou S, Daida H, Dohi S, Egusa G, Hiro T, Hirobe K, lida M, Kihara S, Kinoshita M, Maruyama C, Ohta T, Okamura T, Yamashita S, Yokode M, Yokote K; Japan Atherosclerosis Society (JAS). Comprehensive risk management for the prevention of cardiovascular disease: executive summary of the Japan Atherosclerosis Society (JAS) guidelines for the diagnosis and prevention of atherosclerotic cardiovascular diseases in Japan - 2012. J Atheroscler Thromb. 2013;20:603-15.

20. Saito E, Okada T, Abe Y, Kazama M, Yonezawa R, Kuromori Y, Iwata F, Hara M. Non-high-density Lipoprotein Cholesterol Levels in Japanese Obese Boys with Metabolic Syndrome. J Atheroscler Thromb. 2016;23:105-11.

21. Huang J, Parish R, Mansi I, Yu H, Kennen EM, Davis T, Carden D. Non-high-density lipoprotein cholesterol in patients with metabolic syndrome. J Investig Med. 2008;56:931-6.

22. Sharma B, Saha A, Dubey NK, Kapoor K, Anubhuti, Batra VV, Upadhayay AD. Endothelial dysfuntion in children with idiopathic nephrotic syndrome. Atherosclerosis. 2014;233:704-6.

23. Carpenter SL, Goldman J, Sherman AK, Selewski DT, Kallash M, Tran CL, Seamon M, Katsoufis C, Ashoor I, Hernandez J, Supe-Markovina K, D'alessandri-Silva C, DeJesus-Gonzalez N, Vasylyeva TL, Formeck C, Woll C, Gbadegesin R, Geier P, Devarajan P, Smoyer WE, Kerlin BA, Rheault MN. Association of infections and venous thromboembolism in hospitalized children with nephrotic syndrome. Pediatr Nephrol. 2019;34:261-67.

24. Stabouli S, Chrysaidou K, Kupferman JC, Zafeiriou DI. Neurological complications in childhood nephrotic syndrome: A systematic review. Eur J Paediatr Neurol. 2019;23:384-91.

25. Ambrosch A, Müller R, Freytag C, Borgmann S, Kraus J, Dierkes J, Neumann KH, König W: Small-sized low-density lipoproteins of subclass $B$ from patients with end-stage renal disease effectively augment tumor necrosis factor-a-induced adhesive properties in human endothelial cells. Am J Kidney Dis 2002;39:972-84. 
26. Pennathur S, Heinecke JW. Oxidative stress and endothelial dysfunction in vascular disease. Curr Diab Rep. 2007;7:257-64.

27. Santilli F, D'Ardes D, Davì G. Oxidative stress in chronic vascular disease: From prediction to prevention. Vascul Pharmacol. 2015;74:23-37.

28. Paisley KE, Beaman M, Tooke JE, Mohamed-Ali V, Lowe GD, Shore AC. Endothelial dysfunction and inflammation in asymptomatic proteinuria. Kidney Int. 2003;63:624-33.

29. Ivanova EA, Myasoedova VA, Melnichenko AA, Grechko AV, Orekhov AN. Small dense low-density lipoprotein as biomarker for atherosclerotic diseases. Oxidative Med Cell Longev. 2017;2017:1273042.

30. Dobiasova M. "AIP-atherogenic index of plasma as a significant predictor of cardiovascular risk: from research to practice" Vnitrni Lekarstvi. 2006;52:64-71.

31. Mérouani A, Lévy E, Mongeau JG, Robitaille P, Lambert M, Delvin EE. Hyperlipidemic profiles during remission in childhood idiopathic nephrotic syndrome. Clin Biochem. 2003;36:571-4.

32. Zilleruelo G, Hsia SL, Freundlich M, Gorman HM, Strauss J. Persistence of serum lipid abnormalities in children with idiopathic nephrotic syndrome. J Pediatr. 1984;104:61-4.

33. Kniazewska MH, Obuchowicz AK, Wielkoszynski T, Zmudzinska-Kitczak J, Urban K, Marek M, et al. Atherosclerosis risk factors in young patients formerly treated for idiopathic nephrotic syndrome. Pediatr Nephrol. 2009;24:549-54.

34. William A Prescott Jr 1 , Darcie-ann D Streetman, Daniel S Streetman. The Potential Role of HMGCoA Reductase Inhibitors in Pediatric Nephrotic Syndrome. Ann Pharmacother. 2004;38:2105-14.

35. Skrzypczyk P, Panczyk-Tomaszewska M, Roszkowska-Blaim M, Wawer Z, Bienias B, Zajgzkowska M, Kilis-Pstrusinska K, Jakubowska A, Szczepaniak M, Pawlak-Bratkowska M, Tkaczyk M. Long-term outcomes in idiopathic nephrotic syndrome: from childhood to adulthood. Clin Nephrol. 2014;81:16673.

36. Warady BA, Abraham AG, Schwartz GJ, Wong CS, Muñoz A, Betoko A, Mitsnefes M, Kaskel F, Greenbaum LA, Mak RH, Flynn J, Moxey-Mims MM, Furth S. Predictors of Rapid Progression of Glomerular and Nonglomerular Kidney Disease in Children and Adolescents: The Chronic Kidney Disease in Children (CKiD) Cohort. Am J Kidney Dis. 2015;65:878-88.

37. da Silva PM, Duarte JS, von Hafe P, Gil V, de Oliveira JN, de Sousa G. Standardization of laboratory and lipid profile evaluation: A call for action with a special focus in 2016 ESC/EAS dyslipidaemia guidelines - Full report. Atheroscler Suppl. 2018;31:e1-e12.

\section{Tables}

Due to technical limitations, table 1 is only available as a download in the Supplemental Files section.

\section{Figures}




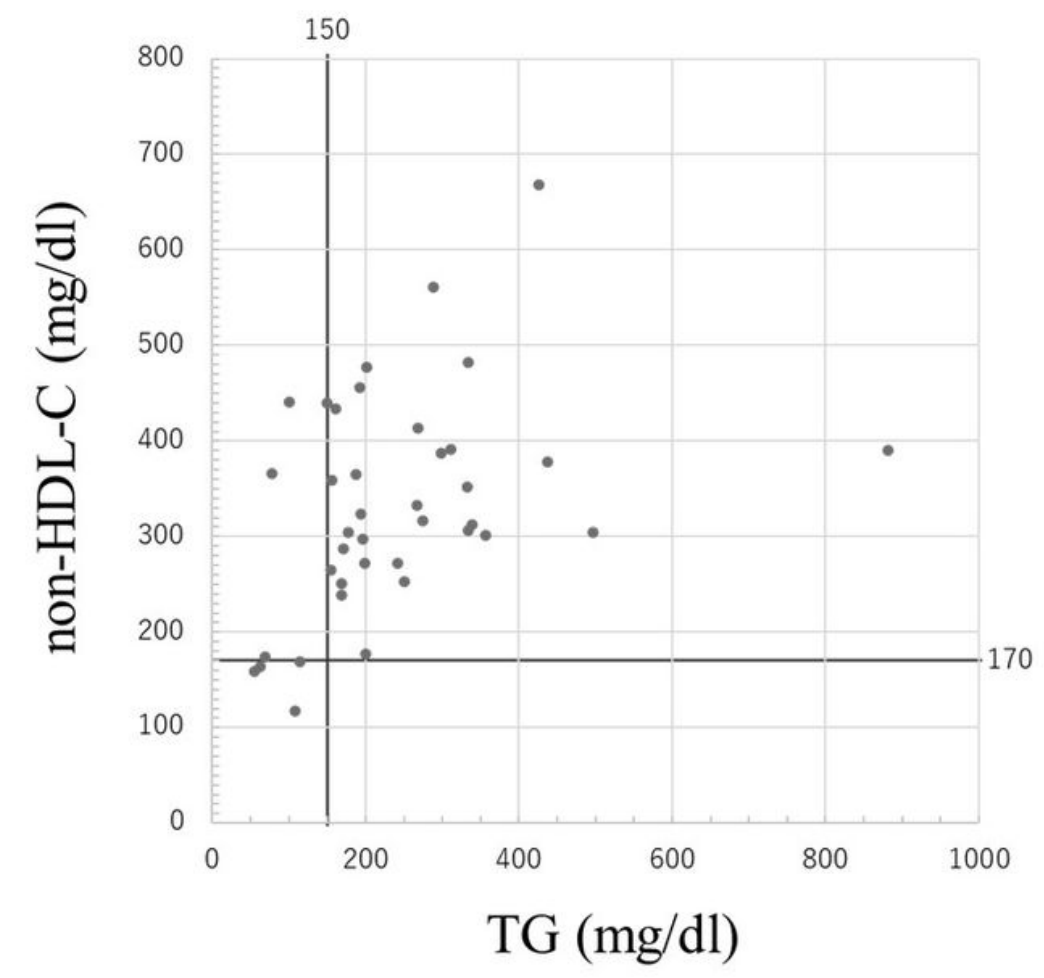

Figure 1

\section{Figure 1}

The alternative LDL window according to non-HDL-C and TG levels The "alternative LDL window" comprises the number and sizes of LDL particles estimated according to plasma non-HDL-C and TG levels. Subjects were divided into the following four groups: normal (non-HDL-C $<170$ and TG $<150$ $\mathrm{mg} / \mathrm{dL}$ ), hyper-TG (non-HDL-C $<170$ and TG $\geq 150 \mathrm{mg} / \mathrm{dL}$ ), hyper-non-HDL (non-HDL-C $\geq 170$ and TG $<$ $150 \mathrm{mg} / \mathrm{dL}$ ), and hyper-TG/-non-HDL (non-HDL-C $\geq 170$ and TG $\geq 150 \mathrm{mg} / \mathrm{dL}$ ). Of 39 patients, 32 (82\%) presented with hyper-TG/-non-HDL levels, and 3 patients (8\%) presented with hyper-non-HDL levels

\section{Supplementary Files}

This is a list of supplementary files associated with this preprint. Click to download.

- Table1.JPG 